\title{
MDCT-based lung volumetry as a prognostic tool—miles to go before we sleep
}

\author{
Harsh Mahajan $^{1} \cdot$ Rohan Shad ${ }^{2}$
}

Received: 27 April 2017 / Accepted: 1 May 2017 / Published online: 17 June 2017

(C) Indian Association of Cardiovascular-Thoracic Surgeons 2017

Pulmonary contusions are the most common parenchymal lung injury following blunt trauma to the chest, and while the management is largely supportive, the clinical course of the disease remains uncertain at best [1]. Multidetector computed tomography (MDCT) of the chest is a useful tool in assessing the severity of pulmonary contusions, but predicting both poor outcomes and the need for ventilatory support have proved to be a challenge.

Studies have previously identified initial $\mathrm{PaO}_{2} / \mathrm{FIO}_{2}$, Injury Severity Score, Revised Trauma Scores, GCS $<12$, and shock or a need for blood transfusions as factors that predict a need for ventilatory support in patients with pulmonary contusions [2]. Traditional chest X-rays tend to lag the clinical course of the disease, and while MDCT can detect pulmonary contusions that are yet to manifest clinically, MDCT on its own has had limited utility as a tool for prognostication [1]. In a study by Millers et al. for example, a small cohort of 49 patients showed that there was no consistent relationship of severity of pulmonary contusion with admission $\mathrm{PaO}_{2} / \mathrm{FIO}_{2}$ [3]. In a study by Deunk et al., the volume of pulmonary contusion was independently associated with a complicated recovery yet was still not predictive of the need for ventilatory support or mortality [4].

The authors of the study published in this issue of the Journal [10.1007/s12055-017-0559-1] have used MDCTbased lung volumetry in an attempt to predict the need for mechanical ventilation in patients presenting with pulmonary contusions. The authors concluded that higher uninvolved

Rohan Shad

rohan.shad@gmail.com

1 Mahajan Imaging, Delhi, India

2 University College of Medical Sciences, Delhi, India lung volumes were associated with an uneventful recovery. Interestingly, advancing age was also associated with an increase in volumes of affected lung tissue. It is unclear whether advancing age contributes more to poor outcomes, or whether it truly is the volume of involved pulmonary tissue that independently predicts this. The lack of a robust multiple regression analysis makes it difficult to come to any conclusions about the predictive nature of lung volumetry alone. Nevertheless, this study has many strengths: a well-crafted study design, strict inclusion criteria for what constitutes isolated blunt thoracic trauma, and a standardized method for MDCT imaging.

The authors have set a standard for exploratory trauma research and demonstrated that routine MDCT imaging can reliably be done for patients in the setting of an Indian trauma center. Furthermore, what we must learn from this study is that MDCT-based volumetry on its own is unlikely to be very predictive for the need for ventilatory support or mortality. To develop highly predictive prognostic models, it is likely that one would require the inclusion of factors such as age, frailty, trauma scores, and metabolic parameters, in addition to high-resolution MDCT-based lung volumetry. Such models though difficult to create and even more so to validate hold the potential to change the way we approach patients with isolated blunt thoracic trauma. We hope this study encourages more work in the field.

\section{References}

1. Ganie FA, Lone H, Lone GN, et al. Lung contusion: a clinicopathological entity with unpredictable clinical course. Bull Emerg Trauma. 2013;1:7-16.

2. Tyburski JG, Collinge JD, Wilson RF, Eachempati SR. Pulmonary contusions: quantifying the lesions on chest X-ray films and the factors affecting prognosis. J Trauma. 1999;46:833-8. 
3. Miller PR, Croce MA, Bee TK, et al. ARDS after pulmonary contusion: accurate measurement of contusion volume identifies high-risk patients. J Trauma 2001;51:223-228-230.
4. Deunk J, Poels TC, Brink M, et al. The clinical outcome of occult pulmonary contusion on multidetector-row computed tomography in blunt trauma patients. J Trauma Inj Infect Crit Care. 2010;68:387-94. 